\title{
Rapid-kilovoltage-switching dual-energy computed tomography (CT) for differentiating spinal osteolytic metastases from spinal infections
}

\author{
Yuan Yuan, Ning Lang, Huishu Yuan \\ Department of Radiology, Peking University Third Hospital, Beijing, China \\ Correspondence to: Huishu Yuan. Department of Radiology, Peking University Third Hospital, No. 49 North Garden Street, Haidian District, Beijing \\ 100191, China. Email: huishuy@bjmu.edu.cn.
}

\begin{abstract}
Background: Rapid-kilovoltage-switching dual-energy computed tomography (RDECT) is a non-invasive, alternative technique for quantitative diagnosis. This study aimed to investigate the value of RDECT for differentiating spinal osteolytic metastases (SOM) from spinal infections (SIs).

Methods: RDECT was performed on 29 patients with SOM and 18 patients with SIs. Both iodine-based and water-based material decomposition images were generated from the spectral CT scan. The iodine/ water densities of lesions on iodine/water material-decomposition images and the CT attenuation values on traditional CT images were measured three times at different image levels, and the averages were calculated. The lesion-to-muscle ratio (LMR) and lesion-to-artery ratio (LAR) for iodine density measurements were calculated. All parameters were compared between the two groups using the two-tailed Student's $t$-test. A P value $<0.05$ was considered statistically significant. The sensitivity and specificity for differentiating SOM from SIs were determined using receiver operating characteristic curves (ROC).

Results: Iodine density, LMR, and LAR during the arterial phase (AP) and venous phase (VP) were all significantly higher for SOM than for SIs (all $\mathrm{P}<0.05$ ). The water densities and traditional CT attenuation values during the AP and VP were not significantly different between the two groups. For ROC analysis, LAR during the VP $\left(\mathrm{LAR}_{\mathrm{VP}}\right)$ showed the best diagnostic performance, with an area under the ROC curve (AUC) value of 0.862 . When the LARVP was 0.54 , the sensitivity was $82.80 \%$ and the specificity was $77.80 \%$ for differentiating SOM from SIs.
\end{abstract}

Conclusions: RDECT can provide additional information that may be useful for differentiating atypical SOM from SIs.

Keywords: Dual-energy CT; differential diagnosis; spinal tumor; spinal infection

Submitted Feb 22, 2020. Accepted for publication Oct 09, 2020.

doi: 10.21037/qims-20-334

View this article at: http://dx.doi.org/10.21037/qims-20-334

\section{Introduction}

Tumor metastases and infections are common pathologies in the spine. Spinal metastases account for $\sim 50 \%$ of all bone metastases and are particularly common in elderly males. Spinal infections (SIs) comprise $5 \%$ of all bone infections and are being increasingly diagnosed in the growing elderly population (1). Differentiating spinal metastasis from SI is crucial, because treatment and prognosis are very different
$(2,3)$. However, as both conditions present with similar signs and symptoms, such as spinal pain and tenderness, limitation of motion, and neurologic manifestations, differentiation based on clinical features alone may be challenging.

When SI presents with typical imaging features, such as vertebral bone destruction, narrowing of the intervertebral disk space and paraspinal abscess, differentiation from spinal 


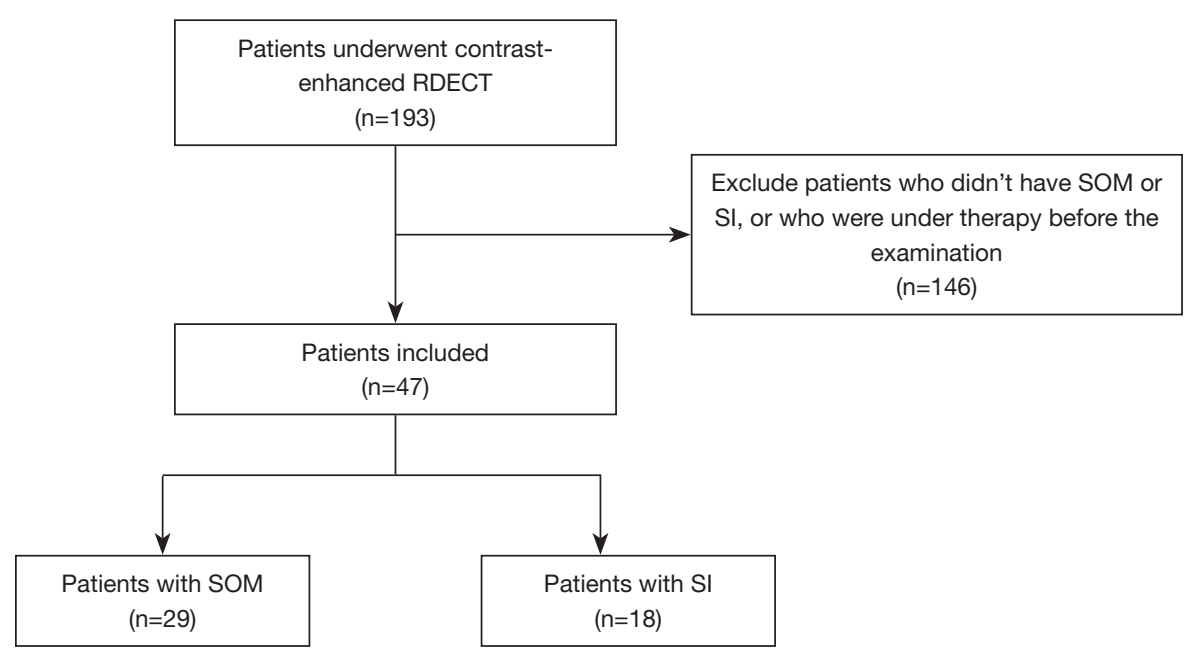

Figure 1 Flow chart of study inclusion criteria.

metastases is easy. However, in select cases, the imaging features associated with SI may be atypical. For instance, there may be destruction of adjacent vertebral bodies without intervertebral disc space involvement, destruction of single vertebral bone, lesions involving only the posterior element, multiple skip lesions in the spine, or absence of abscess formation (3-7). In these instances diagnosis can be difficult.

Rapid-kilovoltage-switching dual-energy computed tomography (RDECT) imaging, a new modality based on rapid switching between 80 and $140 \mathrm{kV}$ tube voltages, has been recently introduced.

With RDECT, a series of quantitative parameters are obtained from iodine-based and water-based material decomposition images and a set of monochromatic images. RDECT imaging has already proved useful in reducing radiation dose and metal artifacts, improving the diagnostic performance of differential diagnoses, and detecting small lesions (8-10). This study aimed to investigate the value of RDECT for differentiating spinal osteolytic metastases (SOM) from SI.

\section{Methods}

Between July 2014 and December 2017, 193 consecutive patients suspected of having vertebral tumors or infections underwent contrast-enhanced RDECT. Of them, 146 patients who did not have osteolytic metastases or infections, who had an unclear diagnosis, or who had undergone therapy before examination were excluded from this analysis. A flow-chart of study inclusion is shown as Figure 1.

Twenty-nine patients (14 men, 15 women; mean age, 58 years; age range, $44-82$ years) had spinal metastases pathologically confirmed by surgery and/or biopsy. The primary tumors included lung cancer $(\mathrm{n}=11)$, breast cancer $(n=7)$, renal cancer $(n=3)$, hepatic cancer $(n=2)$, oesophageal squamous cancer $(n=1)$, gallbladder adenocarcinoma $(n=1)$, intracranial hemangioendothelioma $(\mathrm{n}=1)$, colon cancer $(\mathrm{n}=1)$, cholangiocarcinoma $(\mathrm{n}=1)$, and gastric cancer $(\mathrm{n}=1)$. The sites of the metastases included cervical vertebrae $(n=11)$, lumbar vertebrae $(n=11)$, thoracic vertebrae $(n=4)$, and the sacrum $(n=3)$. Eighteen patients with SI (11 men, 7 women; median age, 64 years; age range, $26-77$ years) had a pathologically confirmed diagnosis of spinal infection. Of the 18 patients, 11 had chronic nonspecific infections and 7 had tuberculosis infections, which were diagnosed by biopsy. The lesions were located in lumbar vertebrae $(n=10)$, cervical vertebrae $(n=6)$, and thoracic vertebrae $(n=2)$.

This study was approved by the ethics committee of our hospital. All procedures were carried out in accordance with the Code of Ethics of the World Medical Association (Declaration of Helsinki). In view of the retrospective nature of the study, the requirement for informed consent was waived.

CT examinations were performed using a GE Discovery CT750 HD scanner (GE Medical Systems, Milwaukee, WI, USA). All unenhanced and contrast-enhanced scans were dedicated spinal CT scans. Unenhanced scans were first performed using the conventional helical scan mode: 
$120 \mathrm{kVp}$ tube voltage, automatic tube current, $0.625 \mathrm{~mm}$ collimation thickness, $5 \mathrm{~mm}$ slice thickness, and 1.375 helical pitch for 13.76-25.85 mGy volumetric CT dose index (CTDIvol). Following this, nonionic contrast medium (1.5 mL/kg body weight, total of $80-100 \mathrm{ML}$, iohexol $350 \mathrm{mg} \mathrm{I} / \mathrm{mL}$; Omnipaque, GE Healthcare) was injected via the antecubital vein at a rate of $3.0 \mathrm{~mL} / \mathrm{s}$ using an automatic power injector (Acist Medical Systems). Dual-energy spectral CT images were obtained in the arterial phase (AP) and venous phase (VP) with delays of 22-24 seconds and 62 to 74 seconds, respectively, from the beginning of the contrast medium injection. The scan range was $1 \mathrm{~cm}$ above and below the lesion. The protocol for dual-energy spectral CT was as follows: tube voltage switching between 80 and $140 \mathrm{kVp}, 550 \mathrm{~mA}$ tube current, $0.625 \mathrm{~mm}$ collimation thickness, 0.8 seconds rotation speed, 1.375 helical pitch, and 16.87 mGy CTDIvol.

Imaging data were reconstructed at a thickness of $0.625 \mathrm{~mm}$ with a standard soft-tissue kernel using dual-energy decomposition algorithms operating in the projection-space domain. The images were sent to an advanced workstation (AW4.5; GE Medical Systems, Milwaukee, WI, USA) and reviewed using the gemstone spectral imaging (GSI) viewer. Circular or elliptical regions of interest (ROIs) were set in the lesions, muscle, and artery on the iodine- and waterbased material decomposition images. The ROIs for the lesions (mean area, $13.67 \mathrm{~mm}^{2}$; range, $3.8-39.16 \mathrm{~mm}^{2}$ ) excluded areas of focal change in large vessels, necrotic tissue, residual bone and prominent artifacts, and included as much of the avidly enhanced areas of the lesions as possible. To ensure consistency, all measurements were repeated three times at different image levels, and the averages were calculated. If the lesion contained relatively more sequestrum or residual bone, three ROIs at the same imaging level were drawn to satisfy the above-mentioned conditions, and averages calculated. The arterial ROIs (37.89 $\mathrm{mm}^{2}$; range, $27-43 \mathrm{~mm}^{2}$ ) were selected from the central two-thirds of the artery, and the ROIs for muscle (109.87 $\mathrm{mm}^{2}$; range, $97-123 \mathrm{~mm}^{2}$ ) were selected from the homogeneous density area of muscle at the same image location as the tumor. All ROIs of the AP were coincident with those of the VP. The measurements were performed by a radiologist with 5 years of experience in musculoskeletal imaging. The iodine/water densities for the lesion, muscle, and artery were automatically obtained on the iodine (water) and water (iodine) material decomposition images using a GSI viewer. The lesion-to-muscle ratio (LMR) and lesionto-artery ratio (LAR) for the iodine density measurements were calculated to allow for comparisons between patients.

According to previous literature (11), the CT values of $70 \mathrm{keV}$ images are similar to those of traditional $120 \mathrm{kV}$ images; therefore, the traditional CT attenuation values of the $\mathrm{AP}$ and $\mathrm{VP}$ were measured on the $70 \mathrm{keV}$ enhanced scanning single-energy images. The ROIs were the same as those on the iodine- and water-based material decomposition images.

Statistical analyses were performed with SPSS (version 20.0) (IBM Corp., Armonk, NY, USA). Continuous variables were expressed as the mean \pm standard deviation. Two-tailed Student's $t$-tests were used to compare the differences in the iodine/water density of lesions, LMR, and LAR during the AP and VP between patients with SOM and SI. For all analyses, statistical significance was set at $\mathrm{P} \leq 0.05$. Receiver operating characteristic curves (ROC) analysis was performed to establish the threshold values and to evaluate the diagnostic ability of each parameter. The Youden index $(\mathcal{F}=$ sensitivity + specificity -1$)$ for differentiating SOM from SI was calculated for all the parameters.

\section{Results}

The iodine-based and water-based material decomposition images are shown as Figures 2 and 3. Table 1 lists the characteristic dual-energy parameters for the SOM and SI groups. Significant differences were measured in iodine density between the SI and SOM groups during the AP $(\mathrm{P}=0.012)$ and $\mathrm{VP}(\mathrm{P}<0.01)$. The LMR in the SI group was significantly lower than that in the SOM group during the AP $(\mathrm{P}=0.019)$ and VP $(\mathrm{P}<0.01)$. The LAR was also significantly lower in the SI group than in the SOM group during the AP $(\mathrm{P}=0.036)$ and $\mathrm{VP}(\mathrm{P}<0.01)$. No significant differences were found in water density between the two groups $(\mathrm{P}>0.05)$ (Table 1). The Hounsfield units were not significantly different between the SI and SOM groups during the AP $(94.94 \pm 22.85$ vs. $110.72 \pm 34.80 \mathrm{HU} ; \mathrm{P}=0.099)$ and VP $(104.65 \pm 27.92$ vs. $116.58 \pm 25.36 \mathrm{HU} ; \mathrm{P}=0.13)$.

Figure 4 includes the ROC curves of the different parameters for differentiating SOM from SI. Table 2 lists the areas under the ROC curve (AUC) values. LAR during the VP $\left(\mathrm{LAR}_{\mathrm{VP}}\right)$ had the largest AUC (0.862). The optimal cut-off values for distinguishing SOM from SI were identified for each parameter (Table 2). For LAR $\mathrm{VP}_{\mathrm{VP}}$ the optimal cut-off was 0.54 , with which the sensitivity was $82.80 \%$ and the specificity was $77.80 \%$. The optimal cut-off for iodine density during the VP was $2.39 \mathrm{mg} / \mathrm{mL}$, which yielded a sensitivity and specificity of $86.20 \%$ and $72.20 \%$, 
A

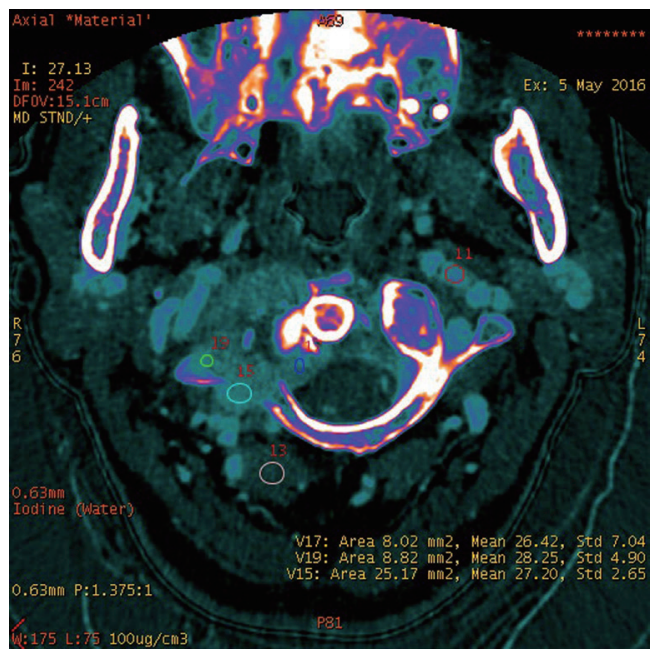

B

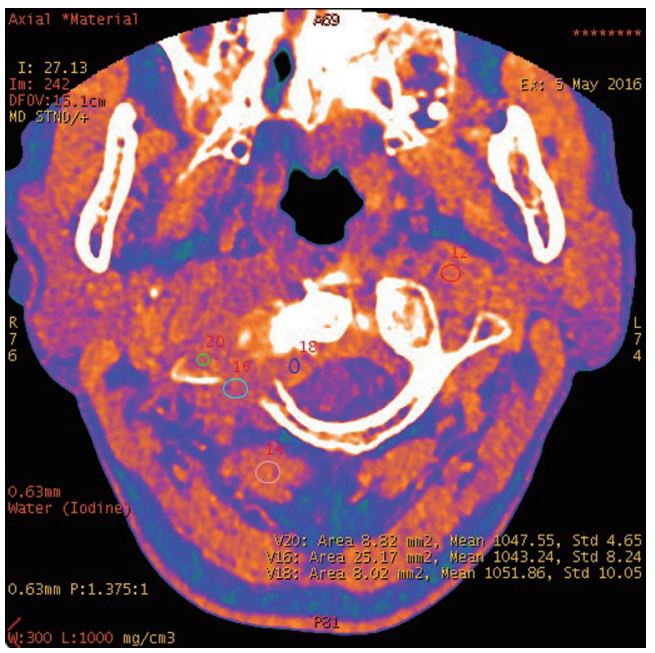

Figure 2 Cervical metastasis of hepatic carcinoma in a 58-year-old woman. The colour coded iodine-based (A) and water-based (B) material decomposition images during the venous phase (VP).

A

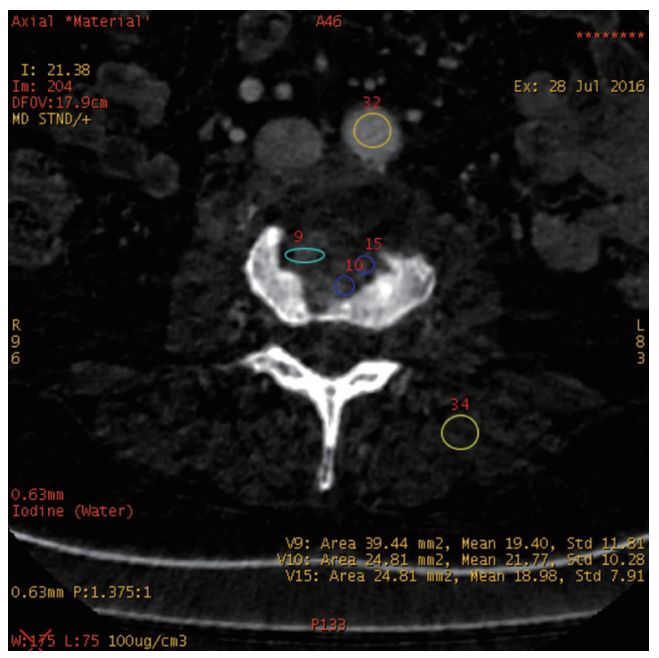

B

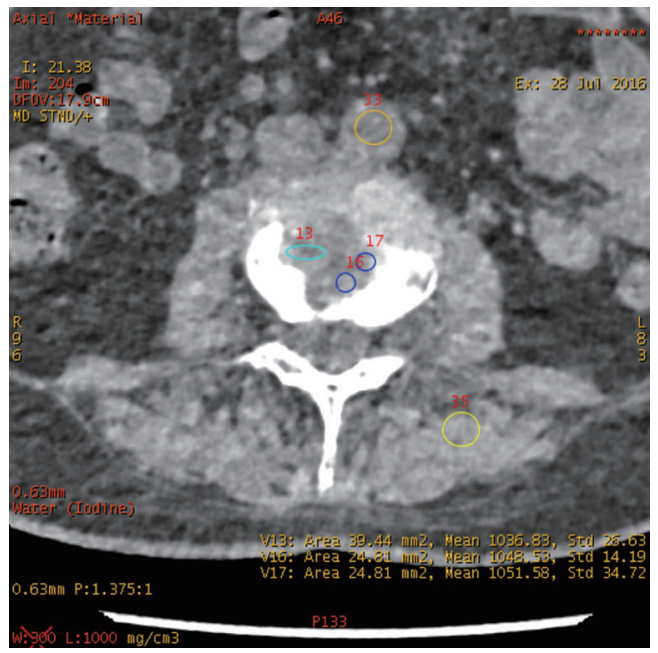

Figure 3 Lumbar tuberculosis in a 76-year-old woman with back pain. Three regions of interest were placed in the lesions, one in the muscle, and one in the artery on the grayscale iodine-based and water-based material decomposition images during the venous phase (VP). Figure $2 A$ shows the average values of iodine density for the lesion $(2.05 \mathrm{mg} / \mathrm{mL})$.

respectively. During the AP, the optimal cut-off for iodine density was $2.57 \mathrm{mg} / \mathrm{mL}$, which yielded a sensitivity of $48.3 \%$ and a specificity of $88.9 \%$.

\section{Discussion}

Spinal metastases and infections are major causes of morbidity and mortality and can have a considerable economic impact on patients. Differentiating between SOM and SI can be difficult, because they present with similar clinical features. The age groups affected by SOM and SI are also similar. For instance, the mean age in our study was 64 years (range, 26-77 years) for patients with SI versus 58 years (range, $44-82$ years) for patients with SOM. 
Table 1 Comparison of different dual-energy computed tomography parameters between spinal infections and spinal osteolytic metastases

\begin{tabular}{|c|c|c|c|c|}
\hline Parameter & SI group & SOM group & $t$ value & $P$ value \\
\hline LMRAP $_{\text {AP }}$ & $6.03 \pm 2.21$ & $8.42 \pm 3.77$ & -2.43 & $0.019^{\star}$ \\
\hline LARAP $_{A}$ & $0.23 \pm 0.07$ & $0.9 \pm 0.10$ & -2.17 & $0.036^{*}$ \\
\hline Water density in AP (mg/mL) & $1,048.05 \pm 20.78$ & $1,047.27 \pm 17.61$ & 0.138 & 0.891 \\
\hline LMRvP & $4.63 \pm 1.59$ & $7.58 \pm 2.63$ & -4.28 & $<0.01^{*}$ \\
\hline LARvp & $0.46 \pm 0.14$ & $0.70 \pm 0.19$ & -4.65 & $<0.01^{*}$ \\
\hline Water density in VP (mg/mL) & $1,037.69 \pm 16.36$ & $1,046.57 \pm 38.71$ & -0.92 & 0.36 \\
\hline
\end{tabular}

*, statistically significant. The data are mean \pm standard deviation. SI, spinal infection; SOM, spinal osteolytic metastases; AP, arterial phase; VP, venous phase; LMR, lesion-to-muscle iodine density ratio; LAR, lesion-to-artery ratio for iodine density.

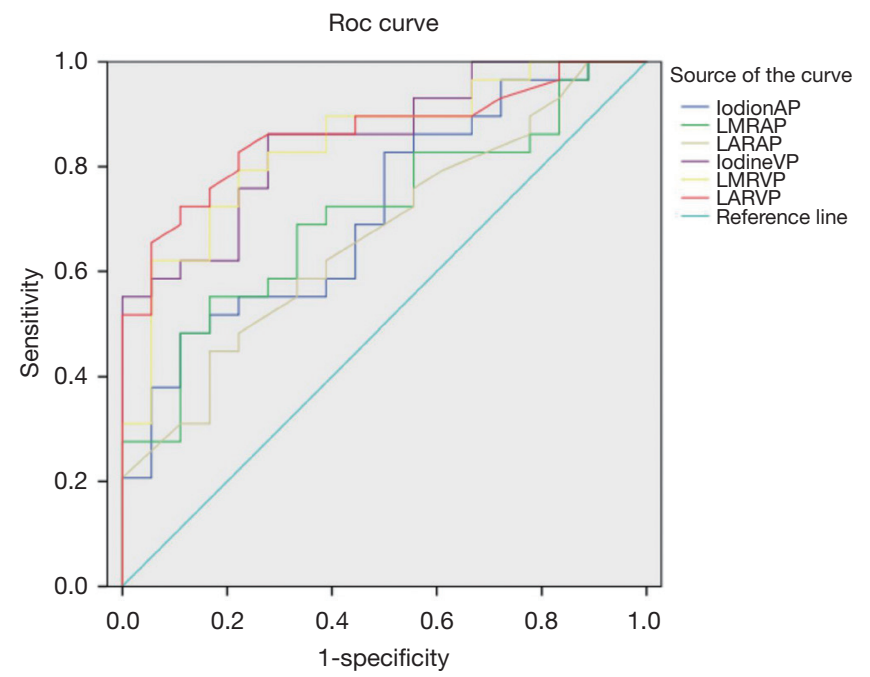

Diagonal segments are produced by ties.

Figure 4 Receiver operating characteristic curves for iodine density, lesion-to-muscle ratio, and lesion-to-artery ratio in differentiating spinal osteolytic metastases from spinal infection.

SI may present with atypical features on imaging. For example, due to discal age-related avascularity, lesions in older patients may be localized in the vertebral body and without associated disc changes (Figure 5) (3). This central type can cause the vertebral body to collapse, mimicking the imaging results of solitary tumor metastasis. In select cases, the infection may spread to several vertebrae via the Batson plexus, creating multiple non-contiguous lesions with an imaging appearance that can be mistaken for multiple metastatic deposits $(3,7,12,13)$. SI may also involve only the posterior element; without vertebral body
Table 2 The threshold values, areas under the receiver operating characteristic curves, sensitivities, and specificities of the parameters assessed

\begin{tabular}{lcccc}
\hline \multirow{2}{*}{ Parameter } & \multicolumn{2}{c}{ Area under } & Threshold & Sensitivity \\
& ROC & value & $\begin{array}{c}\text { Specificity } \\
(\%)\end{array}$ & $(\%)$ \\
\hline lodine density in AP & 0.715 & 2.57 & 48.30 & 88.90 \\
LMRAP $_{\text {LARAP }}$ & 0.707 & 7.45 & 55.20 & 83.30 \\
lodine density in VP & 0.851 & 2.39 & 86.20 & 72.20 \\
LMRvP & 0.670 & 0.25 & 58.60 & 66.70 \\
LARvP & 0.841 & 5.26 & 79.30 & 77.80 \\
\hline
\end{tabular}

ROC, receiver operating characteristic curve; AP, arterial phase; VP, venous phase; LMR, lesion-to-muscle iodine density ratio; LAR, lesion-to-artery ratio for iodine density.

and disc involvement, the imaging appearance is almost indistinguishable from that of a tumor. Finally, SI may occur without the typical formation of paraspinal abscess (14). For the diagnosis of such atypical presentations, a quantitative CT imaging technique is urgently required.

Previous research has reported on the use of spectral CT for differentiating malignant vertebral tumors from non-malignant lesions and for distinguishing osteolytic metastases from Schmorl nodules $(15,16)$. Jans et al. reported that dual-energy CT can visualize bone marrow edema lesions of the hand and wrist in patients with active rheumatoid arthritis, with magnetic resonance imaging (MRI) as a gold standard (17). Fuchs et al. (18) reported that in the assessment of bone marrow, dual-energy CT was less susceptible to metal artifacts than MRI and resulted in 


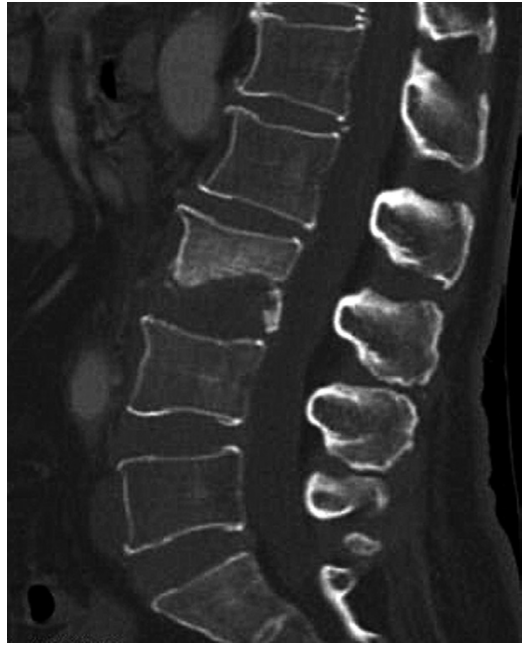

Figure 5 The same patient as Figure 2. Sagittal computed tomography images at $70 \mathrm{keV}$ show osteolytic bone destruction in the L3 vertebral body but no intervertebral disc involvement.

improved visualization of vertebral edema in the vicinity of fused vertebral bodies. Pan et al. reported (19) that the fast $\mathrm{KV}$-switching RDECT HAP-water decomposition technique had an excellent diagnostic performance for identifying acute and chronic vertebral compression fractures in visual and quantitative analyses. However, the usefulness of single-source dual-energy CT imaging for distinguishing between SOM and SI has yet to be investigated.

The X-ray attenuation of a material is determined by the photoelectric and Compton scattering properties (20-22). Each material has a specific energy attenuation curve, and the curve can be represented by the linear combination of two other materials. If the $\mathrm{X}$-ray attenuation of an object is measured on two different spectrums, low $\mathrm{kVp}$ and high $\mathrm{kVp}$, alternating quickly from one view to the next, the attenuation measurements can be mathematically transformed into the density of two materials that is needed to produce the measured attenuation. This process is referred to as material decomposition. Water and iodine are often selected as the basis pair for material decomposition, since they span the atomic number range of materials generally found in medical imaging and can approximate those of soft tissues and iodinated contrast material, thus producing material-attenuation images that can be intuitively interpreted (10). The Discovery CT750 HD obtains virtual monochromatic spectral images at $40-140 \mathrm{keV}$ which are less susceptible to beam-hardening and metal streak artifacts, and allow for more accurate and reproducible attenuation measurements $(23,24)$. Thus, the iodine and water density measured may provide useful quantitative parameters for differential diagnosis.

In the present study, the iodine density, LMR, and LAR in the $\mathrm{AP}$ and VP were significantly higher for SOM than for SI. This is likely due to the relative density of vessels and cells within the lesions, as spinal metastases require angiogenesis to grow and invade. The vessels in metastases are abnormally distributed, and have uneven diameters, wide endothelial junctions, and high permeability $(5,25)$. In comparison, SIs have dilated and relatively mature vasculature. Consequently, the distribution of the contrast agent in the extravascular-extracellular space is more prominent in SOM than in SI. Cell viability and density are also greater in SOM than in SI. Our results are not consistent with those of Tawfik et al. (26), but this may be due to differences in the types of diseases studied and their locations. Tawfik et al. (26) reported on the differences between cervical lymph nodes with acute nonspecific inflammation and lymph nodes with metastases from squamous cell carcinoma, whereas we examined the differences between SI and SOM.

In our study, ROC analysis revealed that $\mathrm{LAR}_{\mathrm{VP}}$ had the best diagnostic value among all the parameters studied; $\mathrm{LAR}_{\mathrm{VP}}$ of 0.54 had a sensitivity of $82.80 \%$ and a specificity of $77.80 \%$ for diagnosing SOM. In a previous study, bone density and water density had a sensitivity of $85 \%$ and $82 \%$, respectively, and a specificity of $97 \%$ and $85 \%$, respectively, for differentiating osteolytic metastasis from Schmorl nodules (15). In another study (24), the optimal threshold $\mathrm{SD}$ value was $68.6 \mathrm{HU}$ (on virtual monochromatic images at $110 \mathrm{keV}$ ) for distinguishing osteoblastic metastases from bone islands in patients with lung cancer; this cut-off provided a sensitivity of $93.0 \%$ and a specificity of $93.3 \%$. In a study on T1-weighted dynamic contrast-enhanced MR imaging, Lang et al. (25) reported that the AUC of $k_{\text {ep }}$ for differentiating between spinal tuberculosis from metastatic cancer was 0.78 . However, the clinical use of T1-weighted dynamic contrast enhanced MR imaging is limited by high costs, long examination time, complexity, MRI artifacts, and some contraindications.

The present study has several limitations. First, this is a retrospective analysis of a small sample, and so these results must be validated in a larger cohort. Second, this study included various SIs, but subgroup analysis could not be performed due to the small number of patients. Third, the SOM group included patients with hyper-vascularized 
and hypo-vascularized metastases. A precise division of primary tumor pathological types will be a priority as we accumulate patients for a future study. Fourth, we did not have data on inter-observer variability. Fifth, since RDECT can only decomposite two materials, calcium was unable to be decomposited when iodine and water were selected as the basis pair for material decomposition, which might have caused measurement bias in lesions with more residual bone. Finally, only the findings of dual-energy spectral CT were studied; the clinical data and radiological features of the patients were not analyzed, mainly because they could not be quantified.

\section{Conclusions}

In conclusion, rapid-kilovoltage-switching dual-energy CT can provide useful information which can help in the differentiation of SOM from SI. The $\mathrm{LAR}_{\mathrm{VP}}$ appears to have the highest diagnostic accuracy.

\section{Acknowledgments}

Funding: This work was supported by National Natural Science Foundation of China (grant numbers: 81871326, 81971578), Natural Science Foundation of Beijing Municipality (grant number: L182054), Peking University Medicine Fund of Fostering Young Scholars' Scientific \& Technological Innovation and the Fundamental Research Funds for the Central Universities (grant number: BMU2020PYB027).

\section{Footnote}

Conflicts of Interest: All authors have completed the ICMJE uniform disclosure form (available at http://dx.doi. org/10.21037/qims-20-334). The authors have no conflicts of interest to declare.

Ethical Statement: This study was approved by the Research Ethics Committee of Peking University Third Hospital, China. The written informed consent was obtained from all patients.

Open Access Statement: This is an Open Access article distributed in accordance with the Creative Commons Attribution-NonCommercial-NoDerivs 4.0 International License (CC BY-NC-ND 4.0), which permits the noncommercial replication and distribution of the article with the strict proviso that no changes or edits are made and the original work is properly cited (including links to both the formal publication through the relevant DOI and the license). See: https://creativecommons.org/licenses/by-nc-nd/4.0/.

\section{References}

1. Babic M, Simpfendorfer CS. Infections of the Spine. Infect Dis Clin North Am 2017;31:279-97.

2. Bilsky MH, Shannon FJ, Sheppard S, Prabhu V, Boland PJ. Diagnosis and management of a metastatic tumor in the atlantoaxial spine. Spine (Phila Pa 1976) 2002;27:1062-9.

3. Mittal S, Khalid M, Sabir AB, Khalid S. Comparison of Magnetic Resonance Imaging Findings between Pathologically Proven Cases of Atypical Tubercular Spine and Tumour Metastasis: A Retrospective Study in 40 Patients. Asian Spine J 2016;10:734-43.

4. Flais J, Coiffier G, Brillet E, Perdriger A, Guggenbuhl P. Atypical presentation of spine bone metastasis in prostate cancer mimicking Pott's disease. Clin Cases Miner Bone Metab 2017;14:239-40.

5. Lang N, Yuan H, Yu HJ, Su MY. Diagnosis of Spinal Lesions Using Heuristic and Pharmacokinetic Parameters Measured by Dynamic Contrast-Enhanced MRI. Acad Radiol 2017;24:867-75.

6. Go SW, Lee HY, Lim CH, Jee WH, Wang YP, Yoo IeR, Kang JY. Atypical disseminated skeletal tuberculosis mimicking metastasis on PET-CT and MRI. Intern Med 2012;51:2961-5.

7. Sezgin B, Atilganoglu U, Yigit O, Ergun SS, Cambaz N, Demirkesen C. Concomitant cutaneous metastatic tuberculous abscesses and multifocal skeletal tuberculosis. Indian J Dermatol 2008;53:149-53.

8. Pessis E, Campagna R, Sverzut JM, Bach F, Rodallec M, Guerini H, Feydy A, Drape JL. Virtual monochromatic spectral imaging with fast kilovoltage switching: reduction of metal artifacts at CT. Radiographics 2013;33:573-83.

9. Lewis M, Reid K, Toms AP. Reducing the effects of metal artifact using high $\mathrm{keV}$ monoenergetic reconstruction of dual energy CT (DECT) in hip replacements. Skeletal Radiol 2013;42:275-82.

10. Lv P, Lin XZ, Li J, Li W, Chen K. Differentiation of small hepatic hemangioma from small hepatocellular carcinoma: recently introduced spectral CT method. Radiology 2011;259:720-9.

11. Krishna S, Sadoughi N, McInnes MDF, Chatelain R, MacDonald DB, Schieda N. Attenuation and Degree of Enhancement With Conventional 120-kVp Polychromatic 
CT and 70-keV Monochromatic Rapid KilovoltageSwitching Dual-Energy CT in Cystic and Solid Renal Masses. AJR Am J Roentgenol 2018;211:789-96.

12. Chang DS, Rafii M, McGuinness G, Jagirdar JS. Primary multifocal tuberculous osteomyelitis with involvement of the ribs. Skeletal Radiol 1998;27:641-5.

13. Zhang L, Wang J, Feng X, Tao Y, Yang J, Zhang S, Cai J. Multifocal skeletal tuberculosis: A case report. Exp Ther Med 2016;11:1288-92.

14. Naim-Ur-Rahman, El-Bakry A, Jamjoom A, Jamjoom ZA, Kolawole TM. Atypical forms of spinal tuberculosis: case report and review of the literature. Surg Neurol 1999;51:602-7.

15. Zheng S, Dong Y, Miao Y, Liu A, Zhang X, Wang B, Ge Y, Liu Y, Wang S. Differentiation of osteolytic metastases and Schmorl's nodes in cancer patients using dual-energy CT: advantage of spectral CT imaging. Eur J Radiol 2014;83:1216-21.

16. Yuan Y, Zhang Y, Lang N, Li J, Yuan H. Differentiating malignant vertebral tumours from non-malignancies with CT spectral imaging: a preliminary study. Eur Radiol 2015;25:2945-50.

17. Jans L, De Kock I, Herregods N, Verstraete K, Van den Bosch F, Carron P, Oei EH, Elewaut D, Jacques P. Dual-energy CT: a new imaging modality for bone marrow oedema in rheumatoid arthritis. Ann Rheum Dis 2018;77:958-60.

18. Fuchs M, Putzier M, Pumberger M, Hermann KG, Diekhoff T. Acute vertebral fracture after spinal fusion: a case report illustrating the added value of single-source dual-energy computed tomography to magnetic resonance imaging in a patient with spinal Instrumentation. Skeletal Radiol 2016;45:1303-6.

19. Pan J, Yan L, Gao H, He Y, Zhong Z, Li P, Zhang Y, Guo Y, Liao L, Zhou S, Zhang K. Fast kilovoltage (KV)-switching dual-energy computed tomography hydroxyapatite (HAP)water decomposition technique for identifying bone marrow edema in vertebral compression fractures. Quant Imaging Med Surg 2020;10:604-11.

20. Avrin DE, Macovski A, Zatz LE. Clinical application of Compton and photo-electric reconstruction in computed tomography: preliminary results. Invest Radiol 1978;13:217-22.

21. Dilmanian FA. Computed tomography with monochromatic $x$ rays. Am J Physiol Imaging 1992;7:175-93.

22. Silva AC, Morse BG, Hara AK, Paden RG, Hongo N, Pavlicek W. Dual-energy (spectral) CT: applications in abdominal imaging. Radiographics 2011;31:1031-46; discussion 1047-50.

23. Matsuda I, Akahane M, Sato J, Katsura M, Kiryu S, Yoshioka N, Kunimatsu A, Ino K, Ohtomo K. Precision of the measurement of CT numbers: comparison of dualenergy CT spectral imaging with fast $\mathrm{kV}$ p switching and conventional CT with phantoms. Jpn J Radiol 2012;30:34-9.

24. Dong Y, Zheng S, Machida H, Wang B, Liu A, Liu Y, Zhang X. Differential diagnosis of osteoblastic metastases from bone islands in patients with lung cancer by singlesource dual-energy CT: advantages of spectral CT imaging. Eur J Radiol 2015;84:901-7.

25. Lang N, Su MY, Yu HJ, Yuan H. Differentiation of tuberculosis and metastatic cancer in the spine using dynamic contrast-enhanced MRI. Eur Spine J 2015;24:1729-37.

26. Tawfik AM, Razek AA, Kerl JM, Nour-Eldin NE, Bauer R, Vogl TJ. Comparison of dual-energy CT-derived iodine content and iodine overlay of normal, inflammatory and metastatic squamous cell carcinoma cervical lymph nodes. Eur Radiol 2014;24:574-80.
Cite this article as: Yuan Y, Lang N, Yuan H. Rapidkilovoltage-switching dual-energy computed tomography (CT) for differentiating spinal osteolytic metastases from spinal infections. Quant Imaging Med Surg 2021;11(2):620-627. doi: 10.21037/qims-20-334 